WillimczrK, Klaus (Hrsg.): Wissenschaftstheoretische Beiträge zur Sportwissenschaft (Texte, Quellen, Dokumente zur Sportwissenschaft. Bd. 14). Schorndorf: Hofmann 1979. 216 S.; DM 24.80.

ZiEgler, Hans-Jürgen: Leistungsfach Sport. Aspekte der Entwidklung eines neuen Schulfaches. (Beiträge zur Lehre und Forschung im Sport. Bd. 74). Schorndorf: Hofmann 1979. 133 S.; DM 14.80.

\title{
Ausschreibung
}

\section{Lehrgang der Internationalen Olympischen Akademie 1980}

Das Nationale Olympische Komitee für Deutschland wird, wie alljährlich, Teilnehmer zum 20. Lehrgang vom 15. Juni bis 1. Juli 1980 in Olympia entsenden. Der Lehrgang steht unter dem Thema „Von den Olympischen Spielen zum Olympismus“. Er feiert auch das 20jährige Bestehen der Akademie. In seinem Rahmen wird die Olympische Flamme für die Olympischen Spiele in Moskau am 19. Juni im Heiligen Hain zu Olympia entzündet. Die Hödstzahl der Teilnehmer eines Landes ist vom Veranstalter auf fünf Personen beschränkt. Die deutsche Beteiligung an der IOA 1980 wird sich auf die vom NOK getroffene Auswahl beschränken. Die Auswahl der Teilnehmer wird aufgrund der eingesandten Unterlagen vom zuständigen Kuratorium des NOK vorgenommen. An die Bewerber werden folgende Anforderungen gerichtet:

a) Sie sollen Studierende im Alter zwischen 18 und 28 Jahren oder ausnahmsweise Lehrkräfte im Alter bis zu 35 Jahren sein;

b) sie sollen mit folgenden Themen vertraut sein oder sich mit ihnen derzeit intensiv befassen:

„Die Olympischen Spiele der Antike und die olympische Bewegung der Neuzeit", „Der moderne Sport und die Probleme der Sporterziehung ${ }^{\text {; }}$;

c) sie sollen gute Sprachkenntnisse in Englisch und/oder Französisch haben und sich mindestens in einer dieser Sprachen verständigen und Vorträgen in dieser Sprache folgen können;

d) sie sollen möglichst vielseitig Sport betreiben und aktives Mitglied eines Sportvereins in der Bundesrepublik Deutschland sein.

Die Bewerber/innen werden gebeten, einen Personalbogen (erhältlich beim NOK) in doppelter Ausfertigung auszufüllen und ihn bis spätestens 15. Februar 1980 über ihre Ausbildungsstätte beim NOK einzureichen. Ein handschriftlicher Lebenslauf unter Angabe des sportlichen Werdegangs, zwei Lichtbilder sowie ein ausführliches Gutachten des Ausbildungsinstituts sind beizufügen.

Das NOK übernimmt für die von ihm gemeldeten Teilnehmer die Kosten für die Sammelreise und die Unterbringung in Olympia. Sie werden in einem Einführungsseminar, voraussichtlich im Mai 1980, auf die Olympische Akademie vorbereitet. Die Beteiligung an diesem Seminar ist Pflicht. Alles Nähere wird den ausgewählten Teilnehmern rechtzeitig mitgeteilt.

Nationales Olympisches Komitee für Deutschland Otto-Fleck-Schneise 12 6000 Frankfurt am Main 71

Telefon (06 11) 67001 gez. Walter Tröger (Generalsekratär) 